\title{
DAVID CRONENBERG $Y$ EL CUERPO ABIERTO
} Artículo de Reflexión

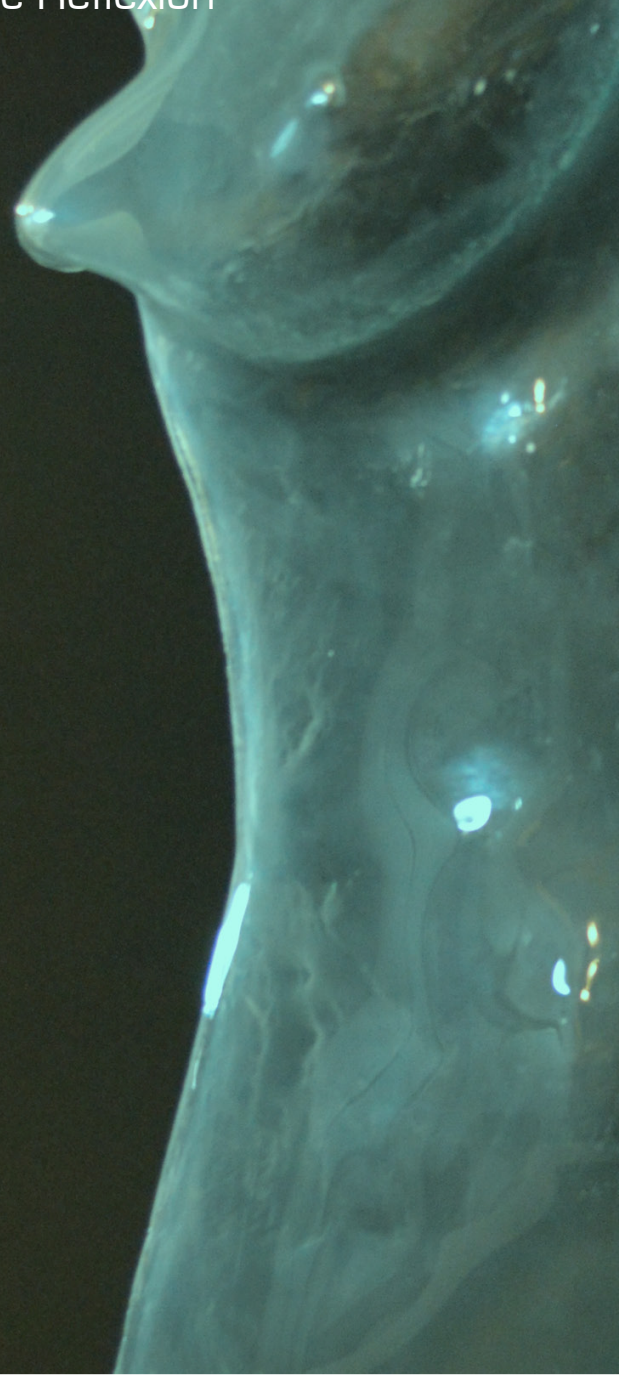

\section{Johanna Rodríguez Ahumada}

\section{Universidad Católica de Colombia / jfrodri4@uc.cl}

Maestra en Artes Plásticas y Visuales de la Universidad Distrital Francisco José de Caldas - Facultad de Artes ASAB [Bogotá, Colombia); Magister en Literatura de la Universidad de los Andes (Bogotá, Colombia); Candidata al Doctorado en Literatura de la Pontifica Universidad Católica de Chile [Santiago de Chile] y docente de la Universidad Católica de Colombia. Este artículo hace parte de la tesis doctoral que actualmente realizo en la Pontificia Universidad Católica de Chile. El título de la investigación en curso es Cuerpos en tránsito. 
RODRíGUEZ J, [2014] David Croenenberg y el cuerpo abierto Calle14, 9 [14] página 106- 117

\title{
DAVID CRONENBERG Y EL CUERPO ABIERTO
}

\section{RESUMEN}

En la sociedad actual, la relación entre el cuerpo y la tecnología ha planteado una de las fisuras más importantes en la concepción de la subjetividad. La enigmática y fascinante relación entre el humano y la máquina, explorada tanto por el cine como por otros [viejos y nuevos] medios en el ámbito de la ciencia ficción, ha planteado la figura del cyborg u organismo cibernético como una criatura híbrida, ambigua, que rompe con la dicotomía naturaleza-artificialidad. ¿Cómo leer los nuevos productos culturales que surgen a raíz de la revolución tecnológica? El objeto de este estudio es Crash (David Cronenberg ,1995), que parte de una interpretación audiovisual de la novela de J.G. Ballard y aborda las fantasías de hibridación del yo con el otro, de lo orgánico con lo inorgánico; en últimas, de cuerpos en tránsito.

\section{PALABRAS CLAVES}

Cuerpo, cyborg, monstruosidad, ciencia ficción, subjetividad.

\section{DAVID CRONENBERG AND THE OPEN BODY}

\begin{abstract}
In today's society, the relationship between the body and technology has raised one of the most important cracks in the conception of subjectivity. The enigmatic and fascinating relationship between human and machine, explored both by film and by other [old and new] media in the field of science fiction, has put forward the figure of the cyborg ["cybernetic organism"] as a hybrid and ambiguous creature that breaks the dichotomy nature-artificiality. How should we read the new cultural products that emerge as a result of the technological revolution? The subject of this study is Crash [1995], directed by David Cronenberg, a visual interpretation of the novel by J.G Ballard that addresses fantasies surrounding the hybridization of the self with the other, of the organic with the inorganic; in short, of bodies in transit.
\end{abstract}

\section{KEYWORDS}

Body, cyborg, monstrosity, science-fiction, subjectivity.

\section{DAVID CRONENBERG ET LE CORPS OUVERT}

\section{RÉSUMÉ}

Dans la société actuelle, la relation entre le corps et la technologie a posé une des fissures les plus importantes dans la conception de la subjectivité. La relation énigmatique et fascinante entre l'homme et la machine, explorée tant par le cinéma comme par d'autres [anciens et nouveaux] medias dans le domaine de la science-fiction, a soulevé la figure du cyborg, ou organisme cybernétique comme une créature hybride, ambiguë, qui brise la dichotomie caractère naturel-artificialité. ¿Comment lire les nouveaux produits culturels qui découlent de la révolution technologique? Le but de cette étude est Crash [David Cronenberg, 1995], qui fait partie d'une interprétation audiovisuelle du roman de JG Ballard et aborde les fantasmes d'hybridation du soi avec l'autre, de l'organique avec l'inorganique; en fin de compte, de corps en transit. 


\section{MOTS CLÉS}

Corps, cyborg, monstruosité, science-fiction, subjectivité.

\section{DAVID CRONENBERG E O CORPO ABERTO}

\section{RESUME}

Na sociedade atual, a relação entre o corpo e a tecnologia concebeu uma das fissuras mais importantes na concepção da subjetividade. A enigmática e fascinante relação entre o humano e a máquina, explorada tanto pelo cinema como por outros [velhos e novos] meios no âmbito da ciência ficção, concebeu a figura do cyborg ou organismo cibernético como uma criatura híbrida, ambígua, que rompe com a dicotomia natureza-artificialidade. Como ler os novos produtos culturais que surgem a raiz da revolução tecnológica? O objeto deste estudo é Crash [David Cronenberg ,1995], que parte de uma interpretação audiovisual da novela de J.G. Ballard e aborda as fantasias de hibridização do eu com o outro, do orgânico com o inorgânico; em últimas, de corpos em trânsito.

\section{PALAVRAS CHAVES}

Corpo, cyborg, monstruosidade, ciência ficção, subjetividade.

\section{KUIRPU PASKASKA DAVID CRONENBERG}

\section{SUGLLAPI}

Kunaura kaugsaipi kami sugrigcha kuirpu ima sugrigcha kunawa imasamika, Runapa kaugsai makinakunawa kawari, atun kawaridirupi imasami ka sugkuna [musu sugkuna Ruku] kawari tukui Sutipa kagsina, [ianga] mailla sutipasina ciburg manchanga waira. Imasata iachangapa imata chikunaka niriagta. ¿lapa iuiaiug ruraskakuna? Kai iuiaska ka crash [David Cronenberg, waranga iskun patsha iskun chunga pichkawata] imasami kallari kawachingapa J.G. Ballard novilapi chagpi sutipasina, chimanda ianga imasa kaugsaskapi chasa.

\section{IMA SUTI RIMAI SIMI:}

Kuirpu, cyborg, ianga kawari, jirurigcha, sutipakagsina, manchangasina. 
En la sociedad actual, la relación entre el cuerpo y la tecnología ha planteado una de las fisuras más importantes en la concepción de la subjetividad y ha sido representada una y otra vez en el imaginario fílmico contemporáneo.

La enigmática y fascinante relación entre el humano y la máquina, explorada tanto por el cine como por otros [viejos y nuevos] medios en el ámbito de la ciencia-ficción, ha planteado la figura del cyborg (cybernetic organism), como una criatura híbrida, ambigua, que rompe con la dicotomía naturaleza-artificialidad. Esos 'organismos cibernéticos' amenazan la identidad única, firme y esencial del humano y han llegado a suscitar reflexiones tan cruciales como las que aparecen en el Manifiesto para cyborgs [1985] de Donna Haraway. Ellos revelan en qué medida el desarrollo tecnológico en estas últimas décadas puede contribuir a un cuestionamiento del pensamiento occidental, de las dicotomías y teleologías que rigen el discurso moderno y que han conducido, entre otras cosas, al establecimiento de la diferenciación entre los géneros y a la regulación de los placeres.

¿Cómo leer los nuevos productos culturales que surgen a raíz de la revolución tecnológica? Crash [1996], película dirigida por director y guionista canadiense David Cronenberg, parte de una interpretación audiovisual de la novela de J.G. Ballard y aborda las fantasías de hibridación del yo con el otro, de lo orgánico con lo inorgánico, de lo masculino con lo femenino, en definitiva, de cuerpos en tránsito.

Lo que la tecnocultura rechaza es el cuerpo real, con sus secreciones, su vulnerabilidad, su impredictibilidad, su fragilidad, su paulatino deterioro y su inevitable deceso. En cambio, el "otro cuerpo", ese que existe solamente como representación, en los medios y que solo podemos aprehender con la mirada nunca fue tan adornado como en este tiempo de diluvio informativo. De esta forma, Susan Sontag en Imagination of Disaster apunta a que el verdadero tema de la ciencia ficción son los desastres que resultan de los esfuerzos del hombre por cambiar el orden natural o modificar su entorno.
David Cronenberg expone el cuerpo como algo que se revela y que repugna. El cuerpo está extendido tecnológicamente, conectado por cables con el mundo y a veces como una "automutilación suicida", como si el sistema nervioso central de esos cuerpos, ya no pudiera depender de los órganos físicos para ser un parachoques protector contra las hondas y flechas mecánicas.

Existe una contradicción de extensión y amputación que se relaciona con una lógica de la tecnología como prótesis, como suplemento, que amenaza con una pérdida, una mutilación. El cuerpo grotesco y barroco delata la materialidad aterradora y obscena de la que está construido, un espacio donde anida la enfermedad, el caos y la degeneración, de donde puede surgir la monstruosidad en cualquier momento.

Tras este concepto de lo monstruoso, Cronenberg expresa temores ancestrales de la humanidad, los miedos al cuerpo mutilado y la transgresión de las fronteras corporales [interiorexterior]. Según el teórico José Miguel Cortés en su libro Orden y Caos: "La sociedad siempre ha tenido monstruos naturales y artificiales, siendo lo monstruoso aquello intolerable que hace nacer en nosotros el horror, la angustia" [1997: 2728].

La nueva carne [expresión que se utiliza especialmente en la película de Cronenberg llamada Videodrome de 1982], se muestra como la mutación del ser humano en criatura material. Pudiendo extenderse más allá de un tema literario o cinematográfico como expone el teórico Pedro Duque en su ensayo "Larga vida a la nueva carne" [1999]:

[...] Estamos asistiendo a toda una fiesta de cambios corporales en los que el tatuaje, el piercing o el body-building son las más inocentes y vulgares de las prácticas, y donde la cirugía radical, la automutilación, los trasplantes y las drogas nos cambian por fuera cambiándonos por dentro.

La Nueva Carne transita, dejando el viejo cuerpo tal como se conoce y convirtiéndose en una vía para entrar en el mundo virtual de la pantalla del 
cualquier potencial y auténtica conexión humana. En el caso de Crash y su esterilidad, vista desde lo anteriormente dicho, se retrata la futilidad de vivir en un mundo donde solo se puede jugar un rol. En este mundo, la homogenización medial de los individuos resulta en una falta de distinción que promueve el aislamiento. Este "vacío de la existencia" conduce a las personas a buscar lo único, interconexiones auténticas y experiencias que nieguen esa pequeñez.

A pesar de los esfuerzos que realizan los personajes, este deseo por lo auténtico y lo significativo no se puede llenar. Todas las interacciones, aun las más privadas, han sido programadas por los medios. La inhabilidad resultante para distinguirse uno mismo y para conectarse de cualquier forma auténtica, incrementa la sensación de aislamiento, lo cual, paradójicamente, refuerza el deseo de comunicación con un otro. La película de Cronenberg examina este deseo y las fuerzas que conspiran para frustrarlo.

En Crash el director canadiense explora la problemática relación de los humanos con los medios tecnológicos. Cronenberg retoma la estética del siglo XX tardío, inspirándose en la novela homónima de James G. Ballard, publicada en 1973. Esta película presenta observadores que experimentan una intensidad tecnológica, entornos urbanos pasajeros que son captados metafóricamente por el tráfico de las grandes y rápidas autopistas. James [James Spader] y Catherine Ballard [Deborah Unger] viven en un rascacielos de Toronto que permite ver el cruce de dos importantes y masivas avenidas. James dirige comerciales de televisión mientras que Catherine está aprendiendo a conducir aeroplanos. Ambos se entregan libremente y sin ambages a relaciones extramaritales. De hecho, la película inicia con una escena sexual seguida por otras dos, un comienzo que Cronenberg diseñó con propósitos de confrontación. Este inicio revela dos personajes que estructuran sus vidas acorde con las narrativas mediáticas [como la pornografía] que, durante la película, ellos adoptan como sus narrativas diarias de "realidad".
La colisión de James con Helen Remington [Holly Hunter] y su esposo [que muere en el choque] fuerza un cambio inesperado en las vidas de estos personajes. Después del accidente, los dos ingresan a un grupo de personas obsesionadas sexualmente con los choques de autos y sus visibles efectos sobre el cuerpo. Esta cohorte está liderada por el enigmático y sobre sexuado Vaughan [Elias Koteas], quien conduce una réplica del convertible Lincoln negro de Kennedy de 1963, y recrea famosos choques automovilísticos [Vaughan admite que el asesinato de John F. Kennedy padre se constituye en un tipo de choque $]^{1}$.

Inicialmente, Vaughan explica sus actos diciendo que trabaja en un proyecto sobre "la reconfiguración del cuerpo humano por la tecnología moderna", pero luego revela que su "proyecto" es para "entender completamente....y vivir" "una psicopatología benevolente”. Esta psicopatología contempla un accidente automovilístico como "una fertilización en vez de un evento destructivo" [esta línea era una que los censores británicos querían cortar]. Unido a Vaughan en este viaje está Colin Seagrave [Peter MacNeil], un anciano que lo ayuda en la puesta en escena de los accidentes y Gabrielle [Rosanna Arquette], una mujer gravemente discapacitada cuyo cuerpo se mantiene unido por correas de cuero, muchos soportes ortopédicos y brazaletes de acero. Los miembros del grupo comparten, como si se tratara de una sala de cine, videos de choques, además de tener sexo en los carros. Estos montajes automovilísticos eventualmente causan la muerte de Vaughan y la asunción de James de su presencia amenazante en la carretera. En la escena final, James fuerza el pequeño carro deportivo de su esposa a salirse de la carretera en un intento no exitoso de compartir la experiencia de choque automovilístico con ella.

1. La obsesión mediática con los detalles del accidente aéreo de John F. Kennedy hijo [julio de 1999] tienta la fascinación humana con el evento catastrófico. Los esfuerzos de la familia Kennedy por remover aspectos del evento del acoso de los medios se convierten en una lucha por su intimidad en medio de un mundo que ha llegado a ser ostensiblemente publicitado. 


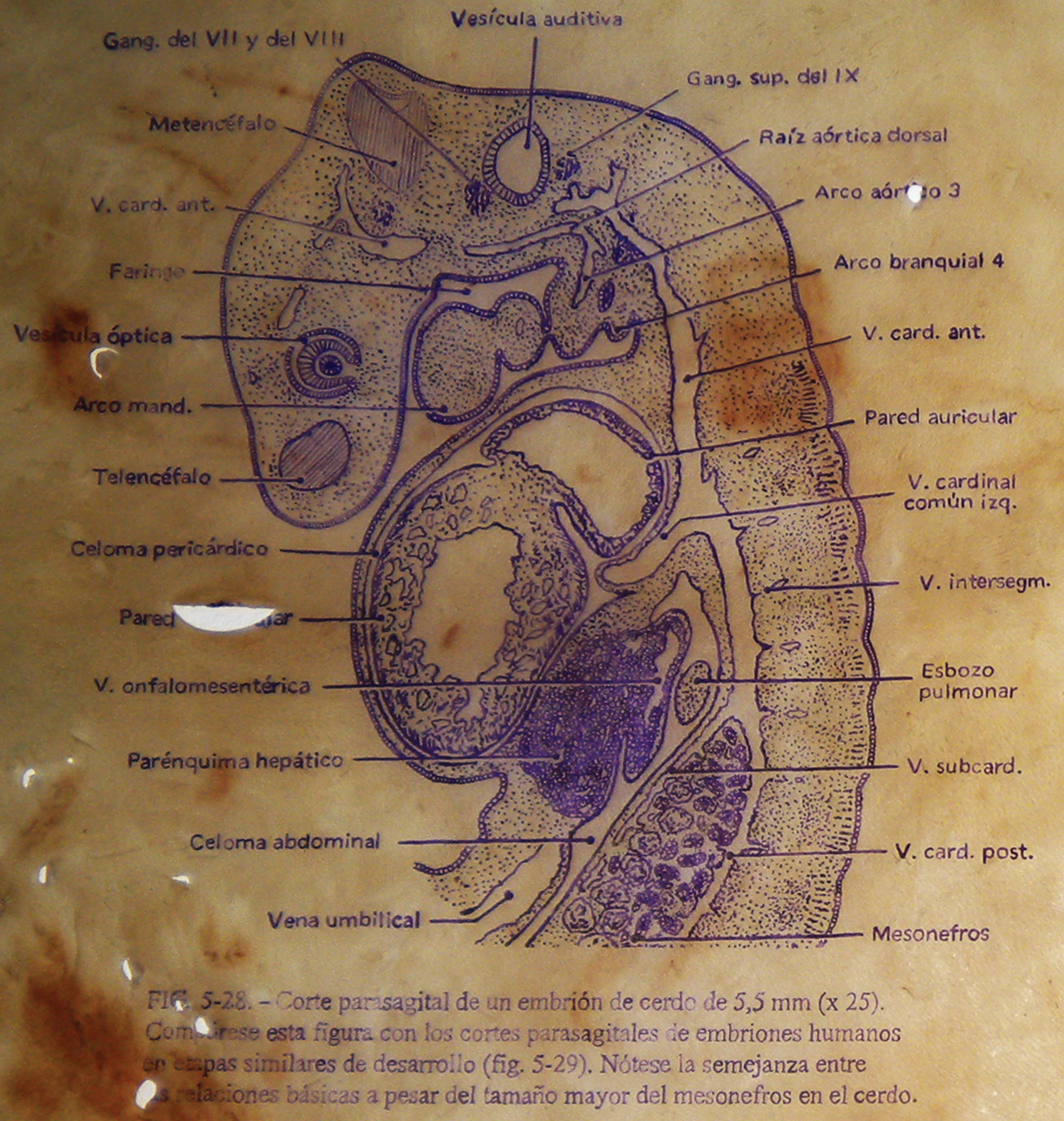

"Los animales asombrados, pasaron su mirada del cerdo al hombre, y del hombre al cerdo; y, nuevamente del cerdo al hombre; pero ya era imposible distinguir quién era uno y quién era otro" Materiales: Violeta de GenciaLos animaties ascmbrados, pasaron su mirad
evamente, del cerdo al hombre; pero ya era ir 




del cerdo al hombre, y del hombre al cerdo;
nposible distinguir quién era uno y quién era otr 
La película crea un mundo revuelto que ofrece un espectáculo posthumano. Crash une individuos con carros que coexisten en una autopista. Todos permanecen en última instancia separados, aun cuando comparten muchos grados de proximidad dentro su rápido mundo. Estos personajes están aislados a pesar de que los une el mismo deseo y simultáneamente están rodeados por otros. Junto con sus encuentros físicos y verbales, configurados por las narrativas mediáticas, no pueden tener muchas interacciones significativas. Lo extensivo de esta invasión de los medios está indicado por el uso de narraciones inspiradas en la pornografía, empleadas por Catherine y James cuando hablan de sus aventuras, y cuyo objetivo reside en la necesidad de inyectarle vida a su sexo marital.

El inesperado accidente de James rompe con la rutina de la pareja y de paso, revela terrenos inexplorados para roles nuevos e intensos sobre el camino. No obstante, esta película ofrece la esperanza de la "realidad", asomando en los auspicios del accidente. Es esta intrusión de lo no inscrito lo que los personajes cortan; como el autor J.G. Ballard [2009] observa: "violence can dismantle that smothering set of conventions that we call everyday reality"2. Mientras tanto, los personajes fetichizan la experiencia del choque y cultivan la nostalgia por accidentes anteriores.

Sentimientos de separación y voyerismo son resaltados por los movimientos de la cámara, las distancias inusuales, y la incapacidad de sutura. Al igual que Hitchcock, Cronenberg juega con el régimen escópico de los espectadores. Esta distancia, la separación, su carencia al no poder involucrarse, la falta de conexión, crean una experiencia de la mirada como solitaria y trastornada. La frialdad de esta experiencia es promovida por el esquema de color que usa Cronenberg: los grises, azules, negros y violetas de la película, enfatizan el detalle frío tanto en los personajes como de los espectadores. Este énfasis es respaldado por la música distante y atonal, desplegada en la obscuridad.

2. "La violencia puede desmantelar ese conjunto asfixiante de convenciones que llamamos realidad cotidiana". [Trad. de Calle 14]
La inhabilidad de encontrar un contacto auténtico en este lustroso mundo resulta en una nostalgia activa por los choques. Los personajes en Crash se mezclan en una repetición nostálgica a nivel tanto de la vida cotidiana [después del choque que sufrió James, él retoma su carro], como al nivel de la fantasía [la reproducción de choques famosos]. En sus intentos de llevar a cabo su deseo por lo real, las víctimas del choque fetichizan el choque en sí mismo y tratan de recapturar la autenticidad en sus sentimientos por medio de la recreación de las escenas de choque, sin toda vez lograrlo. Los personajes no recrean sus propios choques, sino aquellos que se han convertido en legendarios por su puesta en escena en los medios. Las recreaciones elaboradas de los choques de celebridades como James Dean y Jayne Mansfield irónicamente combinan el fetiche con los medios, lo cual niega la autenticidad de los personajes. Cuando los personajes observan sus carros destrozados, los encuentran almacenados en un teatro abandonado. Esto en sí mismo se convierte en una pieza de nostalgia norteamericana, cuando los cines de parqueo al aire libre se asociaban con encuentros sexuales en los carros, apenas comenzada la película.

Por otra parte, en la novela del mismo título, J.G. Ballard juega en un terreno que incorpora los cuerpos físicos con muchas extensiones y componentes, todos ellos en una nueva realidad carnal. Una de esas estructuras significantes presentes en el ámbito cultural es lo que se ha llamado el "cuerpo natural", y la estabilidad del cuerpo humano es como el contenido en esta categoría. Donna Haraway es la donante de este interés en pensar lo múltiple, las identidades cyborg y el deseo de las máquinas, como funciones que dan inicio a nuevas posibilidades, y como caminos a la reimaginación de las relaciones sociales. El proyecto de Haraway no pretende encontrar la unidad de animales o máquinas; ella sospecha de los dualismos y de las síntesis permanentes, incluyendo el dualismo que reina sobre la diferencia entre la naturaleza y la técnica. Alejándose del análisis que ve la técnica y el cuerpo como principios opuestos, Haraway lo refiere como el entendimiento por parte del cuerpo/mente de construcciones en proceso y en tránsito, que permiten repensar las políticas de identidad. 
Emerge no como una fractura final dentro de las subcategorías de la identidad, sino como un entendimiento procesual de la necesidad de conexión que se expresa a sí misma en diferentes energías, las cuales no viajan hacia la unidad, sino que se expresan en múltiples diálogos basados en la diferencia. Ella es consciente de la profundidad de este estado en las políticas de la identidad dentro de los ámbitos contemporáneos, donde las nociones románticas sobre el origen están resquebrajadas.

En su análisis sobre las acciones totalizantes de los proyectos de conocimiento [incluyendo los deseos feministas de un lenguaje común], Haraway apunta a la habilidad dialéctica de la explosión del conocimiento, al desgaste de las certezas, dándonos una bonanza de información y aun así distanciándonos de esas certezas al mismo tiempo. Con toda esa información viene la sospecha, la construcción de un hacer viral que destruye la zona de confort y sumerge al sujeto en proceso en la exploración del tránsito. Haraway abraza esas políticas nómadas que desconocen la identidad, el locus, el foco o la unidad espaciotemporal. Desestabiliza y descentra las historias sobre el origen. Aparte de su afecto por el cyborg y los monstruos emerge en ella la creencia en el poder de lo múltiple, de las historias parciales, delimitando la unidad y el totalitarismo de las estructuras propuestas por los discursos dominantes.

Yo no veo el concepto de "Cuerpo sin Órganos" como una metáfora desencarnada. Artaud y Deleuze/Guattari emplean la frase para hablar sobre la amalgama entre metáfora y carne, significación y experiencia. Escribo desde el lugar de la experiencia de las cicatrices que hacen mi cuerpo, marcas de intervenciones médicas y accidentes. Cuando vi Crash, vi tanto la totalidad, lo plano, lo transitorio, un brillo, una visión y una visualidad sensualmente texturada. Los personajes de Cronenberg son jugadores comprometidos en una simulación de nunca acabar [una visión que se une con la lectura de la novela de Ballard]. Al mismo tiempo, los personajes crean momentos de intersubjetividad a través de extraños puntos de conexión: carros, concreto, cicatrices, metal, al igual que caderas y pechos. El cuerpo es versátil en esos encuentros y en esas nuevas configuraciones. Merleau-Ponty habla sobre la relación entre los diferentes sentidos en la Fenomenología de la percepción:

The sight of sounds and the hearing of colors comes about in the same way as the unity of the gaze through the two eyes: in so far as my body is, not a collection of adjacent organs, but a synergetic system, all the functions of which are exercised and linked together in the general action of being in the world, in so far as it is the congealed face of existence ${ }^{3}$. [1962: 234]

Pero, esta "cara congelada de la existencia" no solo necesita ser extendida al esquema del cuerpo de una persona: la película de Cronenberg toma las relaciones entre visión y autoimagen al extremo, creando "sistemas sinérgicos" que son apropiados y encajan en el mundo en su trayectoria hacia la [pos]modernidad. El cuerpo del yo como instrumento de percepción y lugar de recepción es al mismo tiempo el sitio de lo que es percibido: "My body is the fabric into which all objects are woven." (Merleau-Ponty, 1962: 234). Los rituales que se visibilizan en Crash se hacen carne en el mundo y se extienden en el cuerpo. Nosotros no estamos siendo testigos del nacimiento de una mónada del yo, o, por el hecho de que estamos atestiguando (el compromiso de la textura nemónica], se está alcanzando un entramado de auto-extensión que permite la inclusión de un yo diferente, un otro con el que mezclarse. Finalmente, el dolor hace parte de esa experiencia. Prestar especial atención a los efectos del lenguaje, no solo a nivel social sino también en el plano de la epistemología y la subjetividad, podría ayudar a avanzar hacia el lenguaje abierto del tránsito. En mi lectura, la carne no se convierte en otro idioma deseado, sino en su tránsito incesante.

3. "La visión de los sonidos y la audición de colores se produce en la misma forma que la unidad de la mirada a través de los dos ojos: en la medida en que mi cuerpo es, no una colección de órganos adyacentes, sino un sistema sinérgico cuyas funciones se ejercitan y se conectan entre sí en la acción general de estar en el mundo, en la medida en que este es la cara congelada de la existencia”. [Trad. de Calle 14] 
La yuxtaposición de las palabras cuerpo/cicatriz no tiene sentido para mi experiencia fenomenológica (ya que el dolor y las cicatrices son parte de mi cuerpo, al igual que mis sensaciones]. En mi forma encarnan estas palabras, que por supuesto llevan a identificar y seguir de forma productiva las cosas y se convierten en parte de un continuo de sensaciones que rodean mi existencia en el mundo. Aquí, la carne no se convierte en la falta y pérdida, sino que forma parte de una plenitud de puntos de acceso en una visión liminal de [inter] subjetividad y tránsito continuo.

\section{Referencias}

Ballard, J.G. [2009]. Entrevista con Graeme Revell, en RE/Search 8/9 (1984). Disponible en http://www.v-search.net/books/ballexc4.shtml.

Baudrillard, Jean [1991]. "Two Essays: 1. Simulacra and Science Fiction. 2. Ballard's Crash," translated by Arthur H. Evans. Science-Fiction Studies 18 [3]: 309- 20.

Blakesley, David [1992] "Eviscerating David Cronenberg", en Enculturation. Vol 2. No 1. Fall: 7. 1998. Acceso: 9 feb. www.uta.edu/huma/enculturation/2_1/blakesley/

Cortés, José Miguel G. [1997]. Orden y Caos. Un estudio sobre lo monstruoso en el arte. Colombia: Anagrama. Barcelona: Argumentos.

De Beauvoir, Simone [1974]. The Second Sex. New York: Vintage.

De Lauretis, Teresa [2010]. "Becoming Inorganic: Cronenberg's eXistenZ, Virtuality and the Death Drive", en Freud's drive: Psychoanalysis, Literature and Film, NewYork: Palgrave Macmillan.

Duque, Pedro [1999]. "Larga vida a la carne nueva”, en Goremanía 2. Alberto Santos [ed.].

Gombrich, E. H. [1980]. "Standards of Truth: The Arrested Image and the Moving Eye", en The Language of Images [W. J. T. Mitchell. ed.]. Chicago: University of Chicago Press.

Haraway, Donna [1991]. Simians, Cyborgs, and
Women: The Reinvention of Nature. New York: Routledge.

Hegel, G. W. F. [1977]. Phenomenology of Spirit. Oxford: Clarendon Press.

Hoberman, J. [1997]. "Body Work!" Rev. of Crash, en Village Voice. Marzo 25::75.

Kauffman, Linda [2000]. Malas y perversos. Fantasías en la cultura y el arte contemporáneos. Madrid: Cátedra.

Mauss, Marcel [1973]. "Techniques of the Body." Economy and Society 2: 70- 88.

Merleau-Ponty, Maurice [1968]. The Visible and the Invisible. Evanston, III: Northwestern University Press.

[1964]. The Primacy of Perception. Evanston, III.: Northwestern University Press. [1962]. Phenomenology of Perception. London and New York: Routledge.

MacCarthy, Todd [1996]. "Crash", Rev. of Crash, en Variety 363.3. Mayo 20-26:30.

Rayner, Richard [1997]. "Auto Erotica”, Rev. of Crash, en Harper's Bazaar. Abril: 132.

Scarry, Elaine [1985]. The Body in Pain: The Making and Unmaking of the World. Oxford: Oxford University Press.

Sobchack, Vivian [2004].Carnal Thoughts: Embodiment and Moving Image Cultures. Berkeley: University of California Press.

[1992]. The Address of the Eye: A Phenomenology of Film Experience. Princeton, N.J.: Princeton University Press.

[1991].“Baudrillard's Obscenity”, en

Science Fiction Studies 18 [3].

Fragmento de "Los animales asombrados, pasaron su mirada del cerdo al hombre, y del hombre al cerdo; y, nuevamente del cerdo al hombre; pero ya era imposible distinguir quién era uno y quién era otro" Materiales: Violeta de Genciana sobre piel de Cerdo. Daniel Mauricio Navas Navarro. 2011 


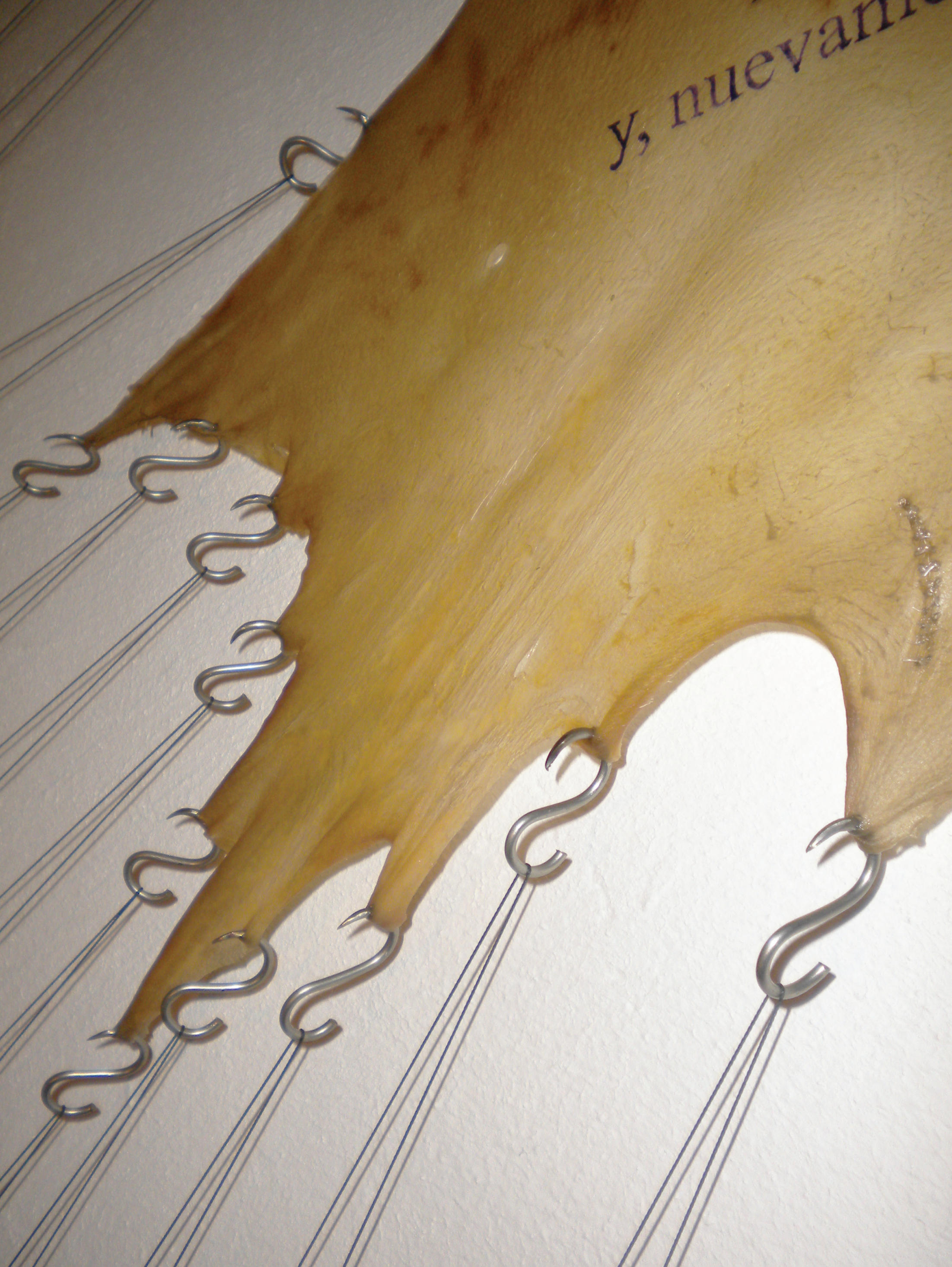

